\title{
IL-I7 is a Potential Therapeutic Target in a Rodent Model of Otitis Media with Effusion
}

\author{
Nanfeng Zhang ${ }^{\prime}, *$, Tingting Qian ${ }^{2, *}$, Shan Sun $\mathbb{D}^{2, *}$, Wei Cao', Zhixian Wang $\mathbb{D}^{\prime}$, Danling Liu², \\ Peifan $\mathrm{Li}^{2}$, Jingfang $\mathrm{Wu}^{2}$, Huawei $\mathrm{Li}^{1,2}$, Jianming Yang' \\ 'Department of ENT, Second Affiliated Hospital of Anhui Medical University, Hefei, Anhui, 23003I, People's Republic of China; ${ }^{2}$ Department of ENT \\ Institute and Otorhinolaryngology, Eye \& ENT Hospital, State Key Laboratory of Medical Neurobiology, NHC Key Laboratory of Hearing Medicine \\ Research, Fudan University, Shanghai, 200032, People's Republic of China
}

*These authors contributed equally to this work

Correspondence: Jianming Yang; Huawei Li, Email JMyang88@I63.com; hwli@shmu.edu.cn

Background: Otitis media with effusion (OME) is a non-suppurative inflammation of the middle ear that is characterized by middle ear effusion and hearing loss. However, the mechanisms of OME are not fully understood. The aim of this study was to determine the function and the mechanism of the IL-17 cytokine in the pathogenesis of OME and to investigate IL-17 as a potential strategy for the treatment of OME.

Methods: In this study, the OME rat model was induced by ovalbumin (OVA) as previously described. The severity of OME was determined with an oto-endoscope, by histochemical analysis, and by acoustic immittance. Kyoto Encyclopedia of Genes and Genomes (KEGG) analysis of RNA-sequencing (RNA-seq) data was carried out to analyze the signaling pathways related to the pathogenesis of OME, which indicated that IL-17 is involved in OME. The anti-IL-17A monoclonal antibody was administrated by nasal drip to block IL-17 to treat OME in the rat model. The rats were finally injected intraperitoneally with the inhibitor of Notch signaling pathway to study the mechanisms of IL-17-induced inflammation. Serum and lavage fluid were collected for the detection of related cytokines, and middle ear tissue was collected for Western blot, quantitative real-time PCR (qRT-PCR), and immunohistochemical and immunofluorescence analysis.

Results: KEGG analysis of RNA-seq data suggested that the IL-17 signaling pathway might be involved in the onset of OME. IL-17 expression was confirmed to be increased in both the serum and the middle ear of the rat model. The monoclonal antibody against IL-17 neutralized IL-17, inhibited the inflammation in the middle ear, and reduced the overall severity of OME in vivo. Furthermore, the Notch signaling pathway was activated upon IL-17 upregulation in OME and was suppressed by IL-17 blockage. However, there was no change in IL-17 expression after Notch inhibitor treatment, which reduced the severity of OME in the rat middle ear.

Conclusion: IL-17 plays a key role in the pathogenesis of the OVA-induced OME rat model. IL-17 induced inflammatory responses via the Notch signaling pathway and targeting IL-17 might be an effective approach for OME therapy.

Keywords: otitis media with effusion, IL-17, anti-IL-17A monoclonal antibody, notch

\section{Introduction}

Otitis media with effusion (OME), also known as exudative otitis media or catarrhal otitis media, is one of the most common ear diseases with the main symptom being hearing loss in young children. ${ }^{1-3}$ OME is characterized by the presence of middle ear effusion (MEE) behind the intact tympanic membrane with no signs or symptoms of an infection. ${ }^{4}$ Some patients are accompanied by mild low-pitched tinnitus and may also be accompanied by mild earache or paroxysmal throbbing pain during the exudative period. ${ }^{5} \mathrm{Up}$ to $80 \%$ of all children experience at least one episode of OME before 10 years of age, and many exhibit long-term complications such as delayed speech, attention deficits, and learning difficulties. Intervention options against OME are largely limited to observation and hearing aids, and patients often require surgical insertion of ventilation tubes through the tympanic membrane. ${ }^{6,7}$ Moreover, OME is considered to 
be one of the most common causes of balance disturbances in young children. ${ }^{8-10}$ Therefore, it is of great significance to understand the pathogenesis of OME in order to develop safe, cheap, and effective therapeutic methods.

OME is one of the main causes of hearing loss, ${ }^{11}$ and the etiology and pathogenesis of OME are multi-factorial. ${ }^{12}$ Although dysfunction of the Eustachian tube is the main etiologic factor in OME, studies have shown that the incidence of OME is also related to factors such as infection, allergies, and immune responses. ${ }^{12,13}$ Children with upper respiratory tract infections, including rhinitis and rhinosinusitis, may initiate and perpetuate inflammation, and this is thought to be the most common predisposing cause of OME. ${ }^{14-16}$ Bacterial biofilms that are involved in the development of pediatric upper respiratory tract infections have also been reported to be related to the pathogenesis of OME. ${ }^{17,18}$ In addition, children with allergic diseases are more susceptible to $\mathrm{OME},{ }^{19-22}$ and the immature function of the immune system is also an important factor in the onset of OME. ${ }^{23}$

The increased obstruction of the local Eustachian tube opening is caused by autoimmune factors, and recent studies have highlighted the physiological function of various immune molecules in the development of OME. IL-4 and IL-5, which play key roles in Th2 responses, have been shown to induce the chronic inflammation of the middle ear in OME, ${ }^{24}$ while IL-4 and IL-13 are involved in the upregulation of mucin metabolism in the middle ear and thus regulate the effects of the chronic inflammation associated with OME. ${ }^{25}$ The MEE samples of OME patients have been shown to contain immune cells such as neutrophils, monocytes/macrophages, NK cells, and T-cells as well as high concentrations of proinflammatory cytokines such as tumor necrosis factor alpha, IL-1 $\beta$, IL-6, and IL-8. ${ }^{26,27}$ IL-17, which is a proinflammatory cytokine secreted by Th17 cells, is also significantly increased in the serum and MEE of children with OME. ${ }^{28}$ However, multiple questions regarding the effect of these immune responses on the onset of OME remain to be answered, including (1) the role of cytokines in the development of OME, (2) the molecular mechanism by which cytokines affect the middle ear in OME, and (3) whether it is possible to develop therapeutic drugs targeting proinflammatory cytokines to treat OME.

OME is characterized by the presence of MEE, which consists of large amounts of mucin secreted by goblet cells. Under normal physiological conditions, mucin only makes up $2 \%$, while solvent accounts for $95 \%$ of the total mucus. Airway mucus is composed of two hydrogels, including the mucus layer and the periciliary layer. The periciliary layer is composed of mucins and other glycoconjugates, ${ }^{29}$ and both layers work together with the ciliated airway cells to protect the airway from pathogenic bacteria. However, under the influence of factors such as air pollution and infection, secretagogues will be excessively secreted. High mucus secretion can impair cilia clearance and local defense functions, leading to increased inflammation. ${ }^{30}$ Muc5ac, which is secreted by goblet cells, is highly upregulated in middle ear inflammation. However, the mechanisms remain unknown.

OME animal models have been developed to investigate the pathogenesis of OME. Histamine-induced early-phase allergic inflammation, which lead to acute OME, was used in many early studies. ${ }^{31}$ Hardy et al then established the latephase allergenic inflammation-induced chronic OME rat model, which is more relevant to the development of OME. ${ }^{32} \mathrm{In}$ this study, an allergen-induced OME rat model was created by sensitization via intraperitoneal injection of OVA followed by intratympanic injection of OVA into the middle ear. Based on the data from RNA-seq, we found that IL-17 was associated with the pathogenesis of OME. Furthermore, anti-IL-17 monoclonal antibody (mAb) treatment by nasal drip neutralized IL-17 and suppressed IL-17-induced inflammation and inflammatory cell infiltration into the middle ear. Moreover, we demonstrated that IL-17 induced the activation of the Notch signaling pathway to enhance the inflammation in the middle ear, thus suggesting a new strategy for the treatment of OME.

\section{Materials and Methods}

\section{Animal Experiments}

Male Sprague-Dawley rats ( 8 weeks old, 160-190 g) were purchased from Airmate Technology (Suzhou, China), and the OVA-induced OME rat model was established as described previously. ${ }^{32}$ All the experimental procedures were performed on the basis of the local Ethics Committee of the Anhui Medical University (which is in accordance with National Laboratory Animal-Guideline for Ethical Review of Animal Welfare (GB/T 35892-2018)). The animal experiments were approved by the Association of Laboratory Animal Sciences at Anhui Medical University (Ethical 
approval number: LLSC20201130). The rats were maintained in the animal facility of Anhui Medical University under specific pathogen-free (SPF) conditions. The animals were grouped in a spacious indoor cage and kept in the animal room under standard conditions ( $12 \mathrm{~h}$ light-dark cycle; temperature $23 \pm 2{ }^{\circ} \mathrm{C}$; moderate humidity $50 \pm 5 \%$ ) with ad libitum access to standard pellets. The Rats were adapted with laboratory conditions for 10 days.

For the first model establishment, the rats were divided randomly into the control group and OME group. During the systemic sensitization phase, rats in the control group and in the OME group were treated with $1.2 \mathrm{mg}$ OVA (A5503; Sigma) (which was dissolved in $0.6 \mathrm{~mL}$ with $14 \mathrm{mg}$ aluminum hydroxide as an immune adjuvant) by intraperitoneal injection on the 1st day after adaptive feeding. On the 8th day, the sensitization injection was repeated. The 15th and 16th days were the stages of immune-challenge. On the 15th day, rats were anesthetized with chloral hydrate solution and rats in the OME group were treated with $0.1 \mathrm{mg}$ OVA by injection into the bilateral middle ear cavity through the posterior lower or anterior lower quadrant of the tympanic membrane using a micro syringe under a microscope. Rats in the control group received PBS. On the 16th day, rats were anesthetized again and the tympanic injection was repeated.

For the second animal model establishment, the rats were randomly divided into the following four groups: wildtype (WT), control (CTRL), OME+Saline, and OME+IL-17 mAb. The rats in the WT group received no treatment. The CTRL group and OME groups were established as described above. In the OME+IL-17 mAb group, $20 \mu \mathrm{L}$ IL-17 mAb, which was diluted with saline to $80 \mu \mathrm{L} / \mathrm{mL}$, was administered by nasal drip after $10 \%$ chloral hydrate anesthesia, which was given 1 hour before the challenge. The rats in the $\mathrm{OME}+$ Saline group were treated with saline by nasal drip. All rats were sacrificed on the 17 th day. Whole blood was collected and centrifuged $\left(3000 \times \mathrm{g}, 10 \mathrm{~min}, 4^{\circ} \mathrm{C}\right)$, and serum samples were obtained for detection of related cytokines. The middle ear tissues were collected and stored at $-80^{\circ} \mathrm{C}$. The rat middle ear was rinsed with $200 \mathrm{ul}$ sterile PBS, and the lavage fluid was kept at $-80^{\circ} \mathrm{C}$ for storage. The middle ear tympanum was fixed in $4 \%$ paraformaldehyde for immunohistochemical and immunofluorescence analysis.

For the third animal model establishment, the rats were randomly separate into four groups: control (CTRL); OME +DMSO; OVA+DAPT (5 mg/kg); and OVA+DAPT (10 mg/kg) group. The $\gamma$-secretase inhibitor DAPT (N-[N-(3, 5-difluorophenacetyl)-lalanyl]-S-phenylglycine t-butyl ester, Sigma-Aldrich, USA) was dissolved in dimethyl sulfoxide (DMSO, Sigma-Aldrich, USA) (vol/vol). The rats were injected intraperitoneally with DMSO or different doses of DAPT $1 \mathrm{~h}$ before each OVA challenge on the 15th day.

\section{HE Staining}

Hematoxylin and eosin staining was performed as previously described. Briefly, fixed middle ear tissues were dehydrated by an ethanol gradient and embedded in paraffin. Sections $(5 \mu \mathrm{m})$ were mounted onto poly-lysine-coated microscope adhesion slides, deparaffinized with xylene, rehydrated with an ethanol gradient, and stained with hematoxylin and eosin according to standard protocols and the manufacturer's instructions.

\section{Acoustic Immittance Map}

The rats were injected intraperitoneally with $10 \%$ chloral hydrate $(330 \mathrm{mg} / \mathrm{kg})$. After general anesthesia, the rat was placed on the experimental table and the external ear canal is disinfected with alcohol, and a probe was inserted into the ear canal to form a closed cavity in the ear canal. By changing the pressure of the external auditory canal, the mobility of the tympanic membrane changes and the dynamic compliance of the middle ear can be observed.

\section{RNA-seq}

\section{RNA Extraction and Library Preparation}

The transcriptome of the middle ear tissues was evaluated by RNA-seq. The tissues were lysed with TRIzol (Invitrogen) and subsequently processed for RNA extraction using the chloroform method. RNA-seq libraries of the middle ear tissue were prepared with TruSeq Stranded Total RNA and a Ribo-Zero Gold kit (Illumina, San Diego, CA, USA). After RNA fragmentation, SPRI beads were used for the size selection of each purification step (Ampure XP, Beckman, USA). An Agilent Bioanalyzer and a Qubit 2.0 fluorometer were used for the quality and concentration analysis of the libraries. The paired-end 150 base pair sequences were generated on an Illumina HiSeq2500 sequencer (Illumina, San Diego, CA, USA). Fastq files of the sequencing data were then obtained and trimmed with Trimmomatic27. The decontaminated 
reads were mapped to the rat reference genome (rn6) using TopHat, and Cufflinks was used for transcript assembly and differential gene expression analysis. The gene expression was assessed by FPKM (Fragments Per Kilobase of transcript per Million fragments mapped), and the genomic features were annotated using the gene transcription information from the RefGene database (NCBI). P-values were adjusted with the Benjamini and Hochberg multiple-testing procedure. The genes with adjusted p-values $<0.01$ were marked as significant. Biological pathways defined by the KEGG (https://www. kegg.jp/) and the Reactome (http://www.reactome.org) databases were used to explore the pathways related to the pathogenesis of OME.

\section{Western Blotting}

One pair of middle ear tissues in a rat were collected as one loading sample. Middle ear tissues were lysed in RIPA buffer, and total lysates were separated with $12.5 \%$ SDS-PAGE and then transferred to a PVDF membrane. The membrane was blocked in blocking buffer then incubated with antibodies against IL-17 and $\beta$-actin for $2 \mathrm{~h}$ at room temperature. After washing in Tris-HCl-buffered salt solution + Tween 20 (TBST) buffer three times for 8 minutes each, the membrane was incubated with the secondary antibody. Finally, the signal was detected using an ECL Western Blotting Substrate. Anti-IL-17 (eBioscience, San Diego, CA, USA), anti- $\beta$-actin (ab8227, Abcam, UK), anti-Notch1 (ab167441, Abcam), and anti-Jagged1 (ab7771, Abcam) were used for the Western blotting according to the manufacturer's instructions.

\section{Quantitative Real-Time PCR}

One pair of middle ear tissues in a rat were collected as one sample. Total RNA was extracted using TRIzol reagent (Invitrogen), and the cDNA was synthesized using a Reverse Transcription Kit from Advansta. qRT-PCR was performed with SYBR Premix Ex Taq II from TaKaRa. Gene-specific primers are listed as follow:

IL-17: forward-(F): 5'-ACAGTGAAGGCAGCGGTACT-3', reverse-(R):5'-GCTCAGAGTCCAGGGTGAAG, Jagged1: F: 5'- GCAACTGTTCCTGAACTCAACT-3', R: 5'-ATCTTTTGGGGTCCGTCAACT-3', Notch1: F: 5'-CTCCAACTG CGACACCAAC-3', R: 5'-CCCTCATAACCTGGCATACA-3', GAPDH: F: 5'-CGGAGTCAACGGATTTGGTCGTAT-3', R: 5'-AGCCTTCTCCATGGTTGGTGAAGAC-3'.

\section{Enzyme-Linked Immunosorbent Assay (ELISA)}

Commercial ELISA kits from CUSABIO (Wuhan, China) were used to measure the concentrations of IL-17 in serum following the manufacturer's instructions. The ELISA plate was coated with anti-rat IL-17 antibody, and the IL-17 in the sample or standard was combined with the coated antibody and the unbound components were washed away. A biotinylated anti-IL-17 antibody and horseradish peroxidase-labeled avidin were then added. The anti-IL-17 antibody bound to the IL-17 that was bound to the coated antibody, and biotin bound specifically to avidin to form an immune complex and unbound components were washed away. The chromogenic substrate TMB was then added, which turns blue under catalysis by horseradish peroxidase and turns yellow after adding stop solution. The OD value at $450 \mathrm{~nm}$ was measured on a microplate reader, and because the IL-17 concentration is proportional to the OD450 value, the IL-17 concentration in the sample was calculated by drawing a standard curve.

\section{Auditory Brainstem Response (ABR) Thresholds}

The subjects were anesthetized using a $10 \%$ chloral hydrate $(330 \mathrm{mg} / \mathrm{kg})$ intraperitoneal injection prior to all experimental sessions. The stimuli were attenuated and filtered (low pass cutoff at $5 \mathrm{kHz}$ ). Stimulus sounds were presented through an electrostatic speaker (TDT EC1) connected to the external ear canal by $10 \mathrm{~cm}$ tubes, thus forming a calibrated, closed system. The rats were placed on a heating pad inside a soundproof booth. Three sub-cutaneous needle electrodes were inserted to record the ABR responses of each mouse, with one at the vertex (non-inverted), one in the mastoid area muscle of the ipsilateral (testing) side (inverted), and one inserted in the muscle posterior to the contralateral pinna as the ground. These electrodes were connected to a Bioamp headstage (HS4 Fiber Optic, TDT). For ABR threshold experiments, the subjects were presented with tonal stimuli in the frequency range of 3 to $48 \mathrm{kHz}$ at various sound levels ( $5 \mathrm{~dB}$ intensity steps) to determine the thresholds. The duration for each ABR stimulus was $5 \mathrm{msec}$ presented at a repetition rate of $21 / \mathrm{s}^{28}$ 


\section{PAS Staining}

PAS staining was used to detect goblet cell proliferation in the middle ear mucosa of OME rats. Paraffin sections were hydrated with an alcohol gradient and then exposed to $0.5 \%$ periodic acid aqueous solution and Schiff reagent in the dark, and then the nuclei were stained with hematoxylin. The images were obtained with a Pannoramic 250/MIDI scanner.

\section{Immunofluorescence Staining}

The middle ear tympanum was dewaxed and rehydrated. The tissue section was heated in a microwave oven for antigen retrieval, and 3\% hydrogen peroxide was used to quench endogenous peroxidase activity. The tissue was evenly covered with BSA for blocking and placed in a humidified incubator with the IL-17 primary antibody (eBioscience, San Diego, CA, USA) overnight at $4^{\circ} \mathrm{C}$. The samples were then incubated with the appropriate secondary antibody corresponding to the primary antibody and then with DAPI for 3 minutes to counterstain the nuclei. Five samples per group were randomly counted under a microscope.

\section{Flow Cytometry Analysis}

Anti-CD45, anti-CD3, anti-CD4, and anti-IL-17A antibodies (eBioscience, San Diego, CA, USA) were used for flow cytometry according to the manufacturer's instructions. For the intracellular staining of IL-17A, cells were stimulated with Cell Stimulation Cocktail (eBioscience) in vitro for $5 \mathrm{~h}$ before the flow cytometry analysis. The cells were then stained for surface markers, and IL-17A was stained with the Intracellular Fixation and Permeabilization Buffer Set (ThermoFisher, Shanghai, China). FlowJo was used to analyze IL-17 expression following the gating strategy shown in Figure S1.

\section{Statistical Analysis}

All data are presented as the mean \pm s.e.m. All statistical analyses were performed with SPSS 19.0. The differences between two groups were analyzed with Student's $t$-test, and the differences between three or more groups were analyzed with one-way ANOVA. $p<0.05$ was considered significant, and $p<0.01$ was considered extremely significant. The results are presented as the mean and the error bars represent the s.e.m., and the numbers of technical or biological replicates are indicated in the figure legends.

\section{Results}

\section{Establishment of OVA-Induced OME in Rats}

As shown in Figure 1A, the modeling was divided into the two stages of systemic sensitization and middle ear immunochallenge. Rats were treated with OVA by intraperitoneal injection for sensitization on the 1st and 8th day after adaptive feeding. On the 15th day and the 16th day, the rats in the OME group were anesthetized with chloral hydrate solution and then injected with OVA into the bilateral middle ear cavity while those in the control group were injected with PBS. On the 17th day, all rats were sacrificed. The establishment of OME was determined with an oto-endoscope, histochemical analysis, and acoustic immittance measurements. As shown in Figure 1B, the tympanic membrane in the CTRL group was transparent. In the OME group, large amounts of MEE were observed in the tympanum membrane that showed otopiesis, and the ear canal exhibited clear radial vascularity. In addition, the mucosa and sub-mucosal tissue were thickened (Figure 1C). The acoustic immittance diagram of the rats in the CTRL group showed type A (Figure 1D), indicating that hearing was normal and there was no evidence of otitis media. The acoustic immittance map of OME rats showed type $\mathrm{B}$ or $\mathrm{C}$ (Figure 1D) and the compliance value was significantly decreased (Figure 1E), indicating that the rats had the typical OME symptoms of MEE and impaired ear function, which confirmed the OME model.

\section{The IL-I7 Signaling Pathway Was Activated in the Middle Ear of the OME Rats}

To investigate the immune responses and the immune molecules that might be involved in the pathogenesis of OME, we analyzed the transcriptome of the middle ear tissues by RNA-seq. KEGG analysis revealed that several signaling pathways related to immune responses were activated in the middle ear of the OME rats, such as the tumor necrosis 


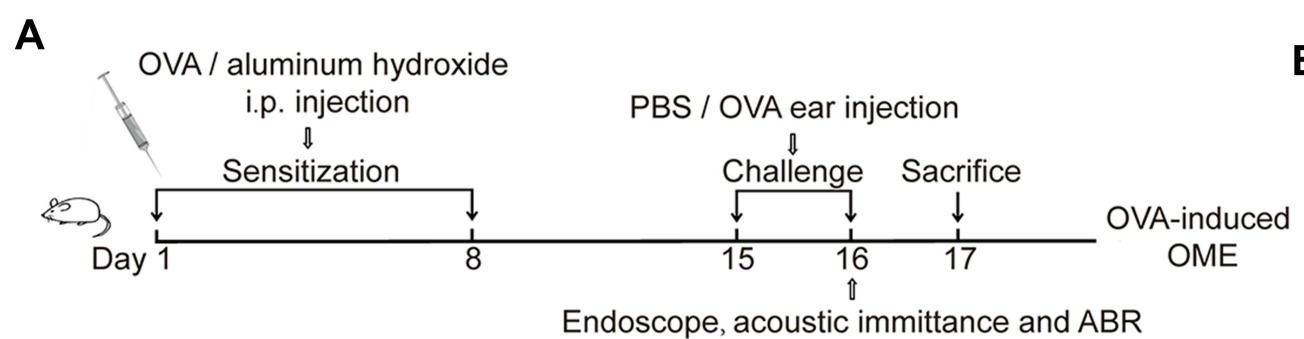

B

CTRL

OME

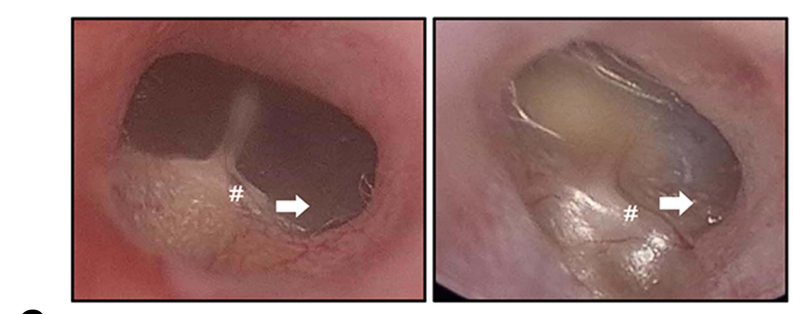

C
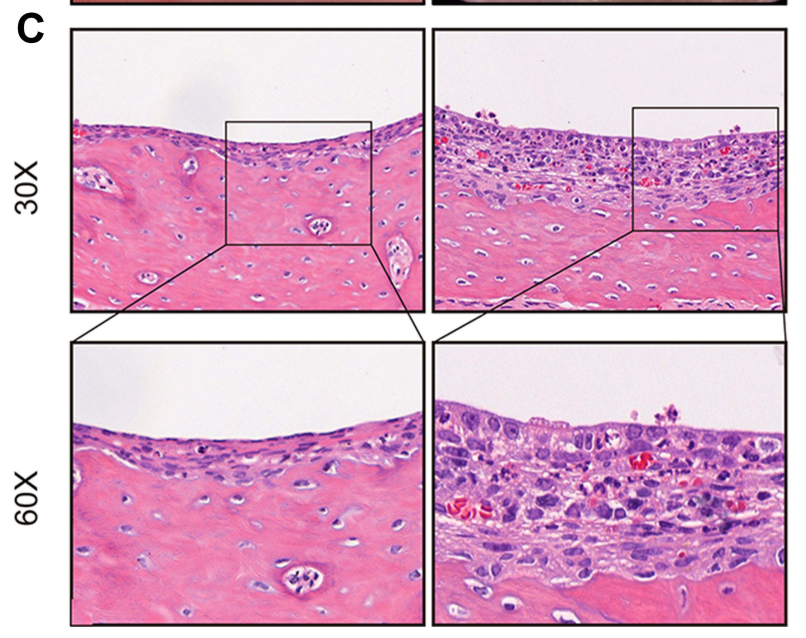

D

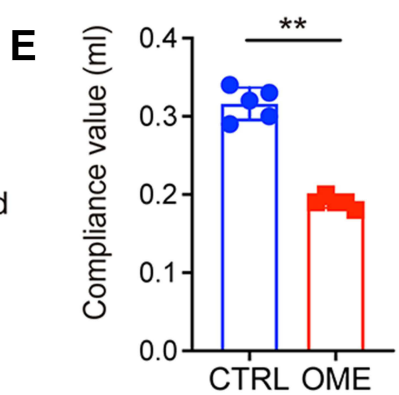

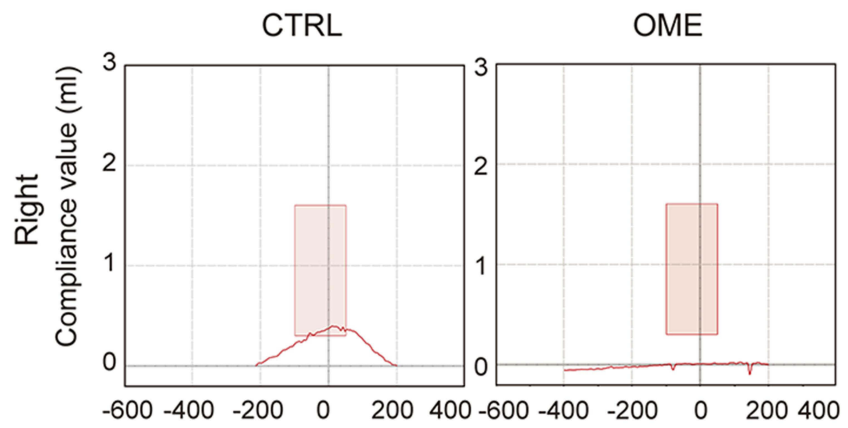

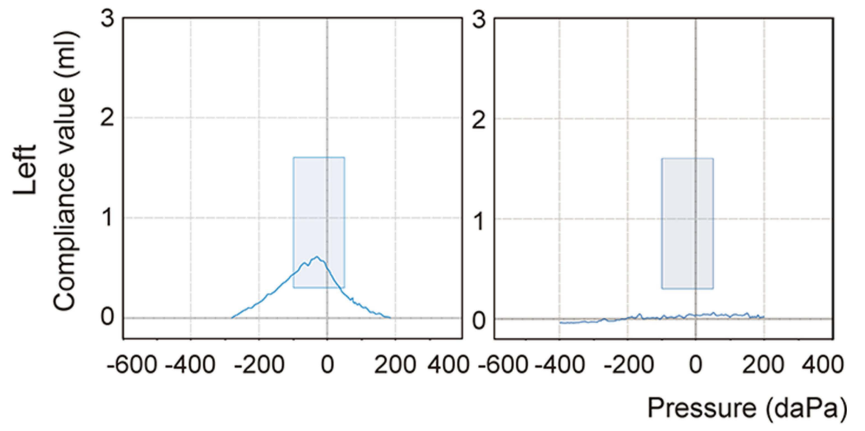

Figure I Evaluation of the OVA-induced OME rat model. (A) The scheme of the experimental OVA-stimulated OME establishment. The rats were sensitized with I.2 mg ovalbumin on day I and day 8 and then challenged by intra-aural injection of OVA or PBS as a control. (B) The morphology of the tympanic membrane viewed through an endoscope showing the secretions (arrow) and the radial vascularity (pound sign). (C) The severity of the middle ear injury was evaluated by HE staining at $30 \times$ and $60 \times$ magnification. (D) The acoustic immittance confirmed the existence of OME in the OME rats. In the control group, both the right ear and left ear were type A or As, which meant that the control group had normal hearing and no otitis media in the ears. In the OME group both the right ear and left ear were type B, which meant that the rats had hearing loss and OME. (E) The statistical analysis of the acoustic immittance according to the compliance values. The error bars represent the s.e.m. of 5 rats per group. $* *$ P $<0.01$.

factor signaling pathway, the Toll-like receptor signaling pathway, the NOD-like receptor signaling pathway, and the IL17 signaling pathway (Figure 2A). The levels of IL-17 and the relevant genes were significantly increased in the OME group (Figure 2B). Quantitative PCR (qPCR) and Western blot demonstrated that IL-17 expression in the middle ear tissues was higher at both the mRNA and protein levels in the OME rats (Figure 2C). Furthermore, the expression of IL17 was upregulated in both the serum and the MEE of the OME rats, as measured by ELISA (Figure 2D).

\section{The Blockage of IL-I7 Signaling Ameliorated OME in the Rat Model}

To determine the functional significance of the IL-17 cytokine in OME, an anti-IL-17 mAb was administrated by nasal drip to block IL-17 prior to the immuno-challenge in the OME rat model (the OME+IL-17 mAb group), while the OME+Saline group was given saline by nasal drip (Figure 3A). The rats in the control group were injected with PBS instead of OVA in the immune-challenge phase (Figure 3A). As shown in Figure 3B, the tympanic membrane of the rats in the control group had little secretion in the tympanic cavity, and the acoustic immittance diagrams showed type A or As diagrams (Figure 3C). As shown in Figure 3B, no obvious tympanic effusion or vascular proliferation was observed in the middle ear of the OME+IL- 
A

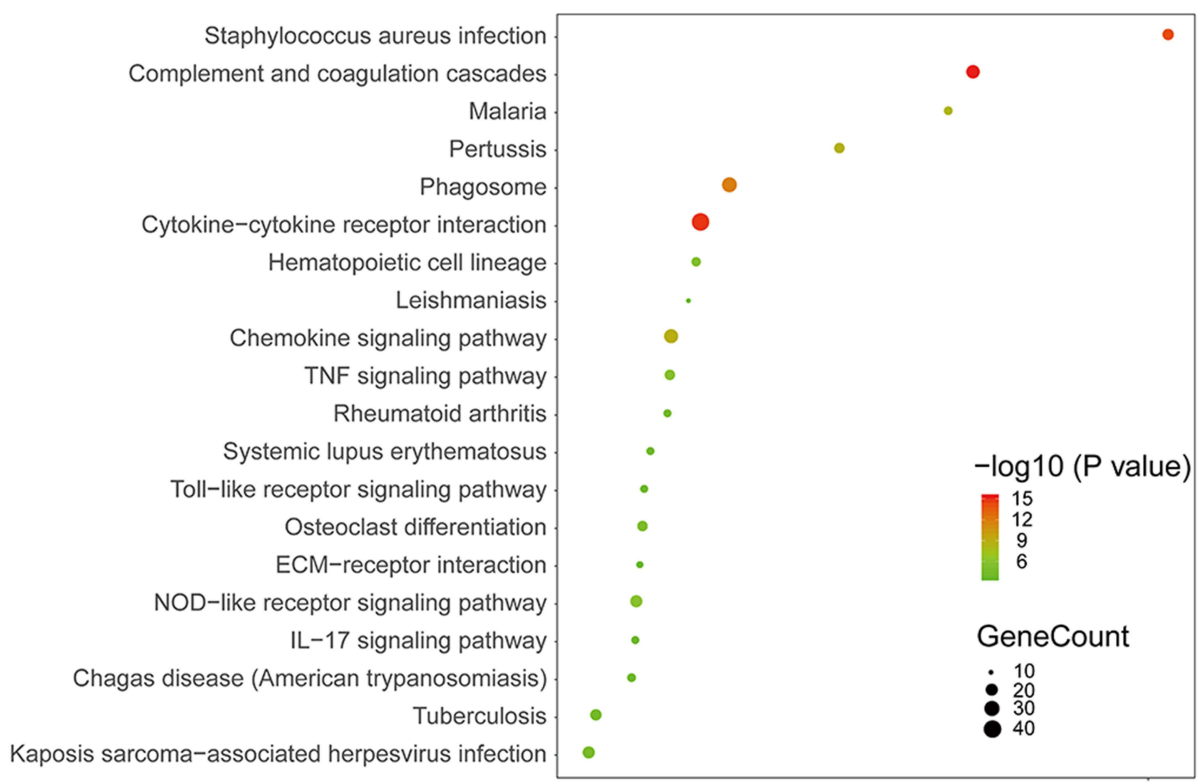

Rich factor

B

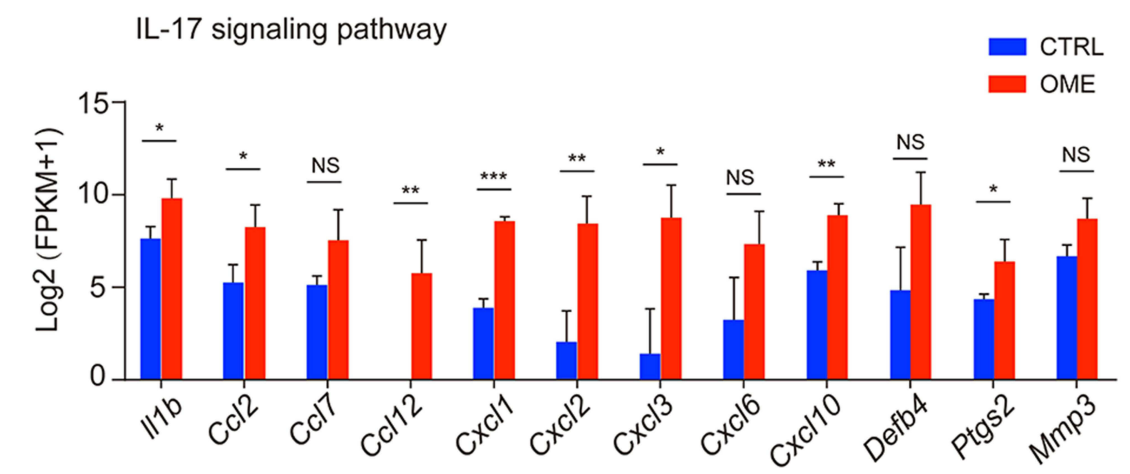

C
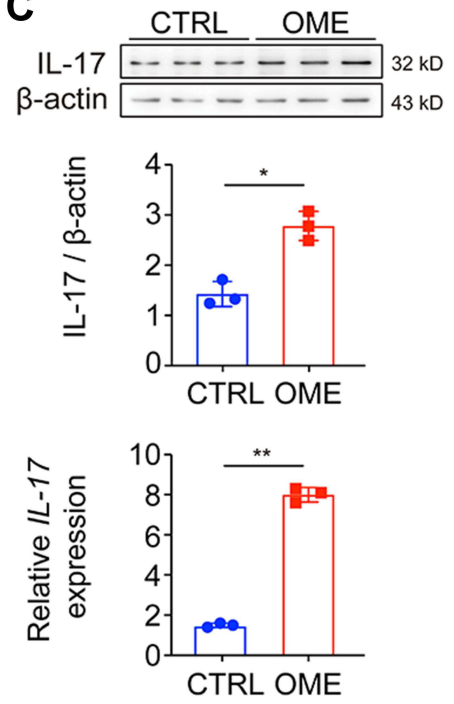

D $\widehat{\bar{\varepsilon}}$
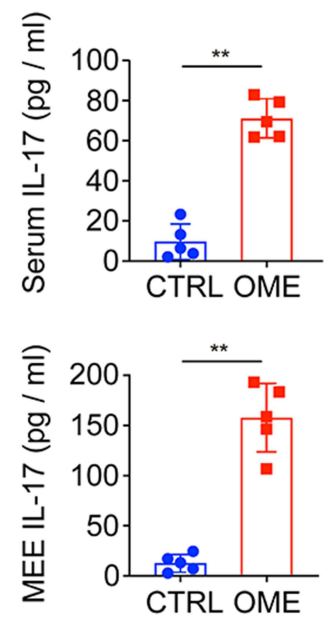

Figure 2 The IL-17 signaling pathway was activated in OME. (A) the KEGG database was used to analyze the pathways using the RNA-seq data. The dot size represents the gene count. The KEGG pathway analysis showed the top 20 enrichments related to OME. (B) The expression levels of genes related to the IL-I7 signaling pathway are shown as log2 $(F P K M+1)$. (C) qPCR and Western blot were used to measure the mRNA and protein expression of II- 17 in the rat middle ear tissue. The error bars represent the s.e.m. of three pairs of ears per group. (D) IL-I7 levels in the serum and MEE of the OME rats were measured by ELISA. The error bars represent the s.e.m. of 5 rats per group. $* \mathrm{P}<0.05$, $* * \mathrm{P}<0.01$, $* * * \mathrm{P}<0.001$. Data are representative of three independent experiments.

Abbreviation: ns, not significant.

$17 \mathrm{mAb}$ group. The acoustic immittance diagrams of the rats in the OME+IL-17 mAb group showed type A or type B diagrams (Figure 3C). Moreover, the rats in the OME+IL-17 mAb group had no ABR threshold elevation compared to PBS-injected controls, while rats in the OME+Saline group had significantly increased ABR thresholds (Figure 3D), implying that IL-17 antibody treatment reduced the overall severity as measured by ABR at $24 \mathrm{~h}$.

We next investigated the pathological effects of the anti-IL-17 mAb on OME. As shown in Figure 4A, the rats in the OME+Saline group showed increased inflammatory cell infiltration and the middle ear mucosa and submucosal tissue became thick compared to the WT and control group. However, the anti-IL-17 mAb treatment significantly reduced the inflammatory cell infiltration into the middle ear (Figure 4A). PAS staining was used to characterize the goblet cells and the mucus that was secreted by the goblet cells in the middle ear. It is obvious that most cells were goblet cells in the OVA group, whereas only a small percentage were detected in the CTRL group, indicating that the goblet cells proliferated and secreted a lot of mucin. However, the anti-IL-17 mAb suppressed the goblet cell proliferation and the mucus secretion (Figure 4B and C). Taken together, these data suggest that anti-IL-17 mAb rescued the OME-induced 
A

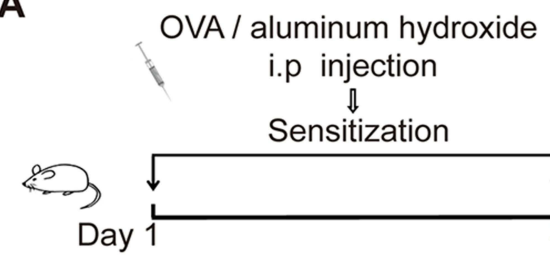

Saline / IL-17 mAb nostril drip before ear injection PBS / OVA ear injection

$$
\sqrt{ }
$$

Challenge
OVA-induced

OME

Endoscope, acoustic immittance and ABR

B
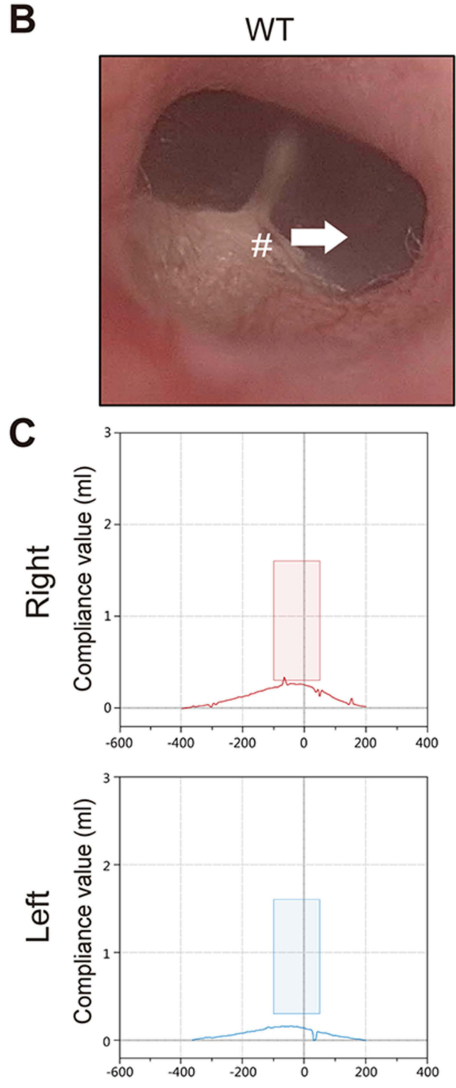

CTRL
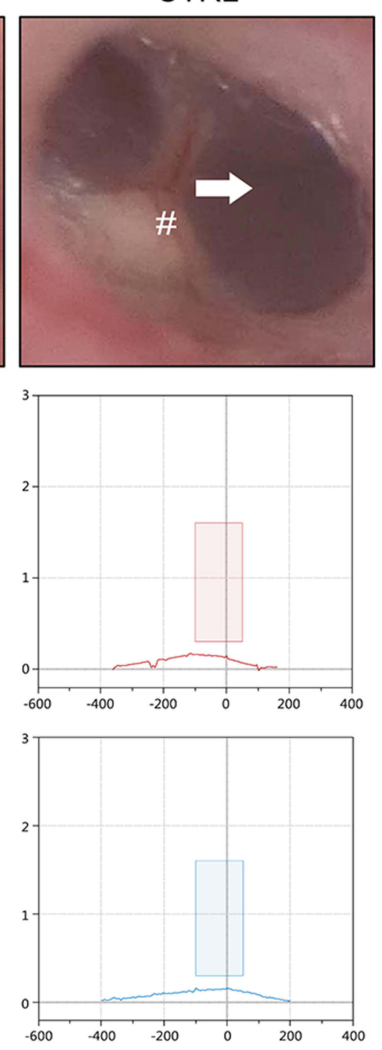

OME + Saline
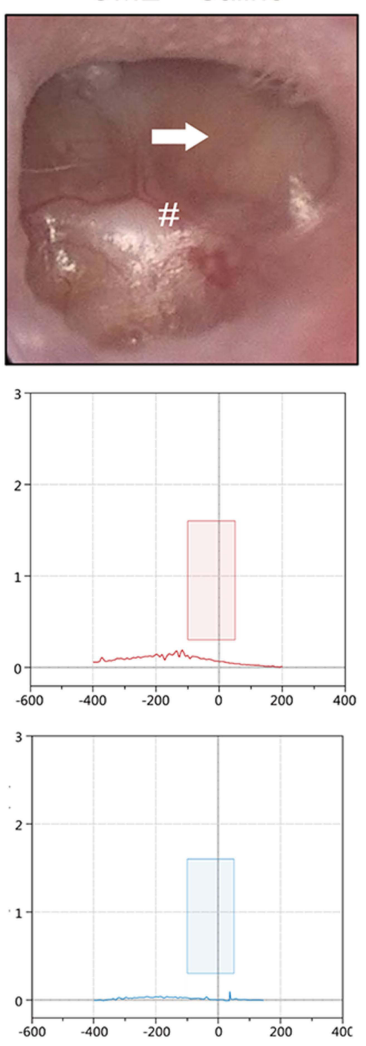

OME + IL-17 mAb
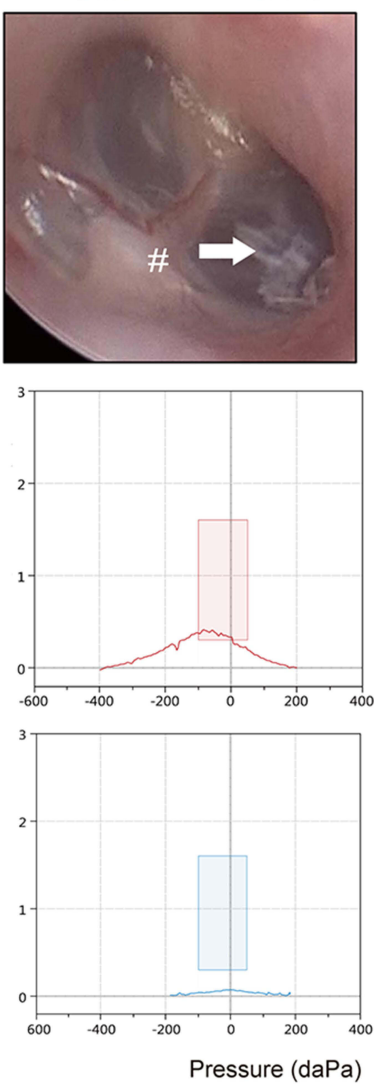

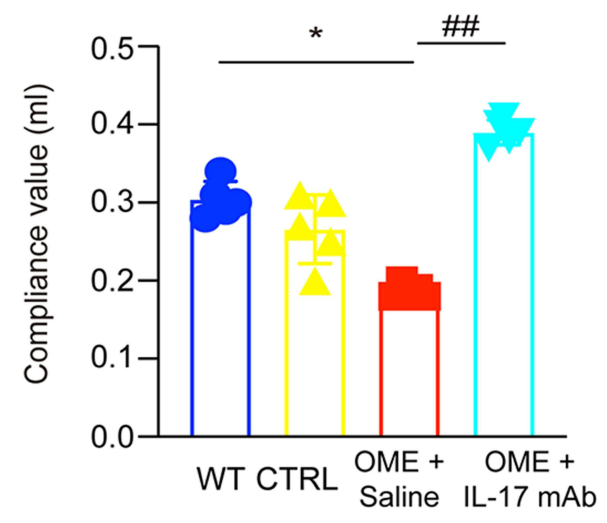

D

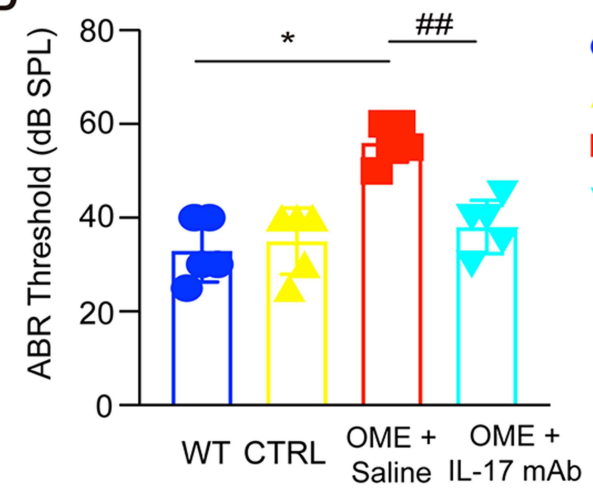

Figure 3 The blockage of IL-I 7 signaling reduced the overall severity of OME in rats. (A) The outline of the experimental OVA-stimulated OME. The rats in the WT group received no treatment. The other rats were sensitized with $1.2 \mathrm{mg}$ OVA on day I and 8 and then challenged on days 15 and I6 with OVA or PBS as a control. The OME+IL$17 \mathrm{mAb}$ group inhaled IL- $17 \mathrm{mAb}$ (diluted to $80 \mu \mathrm{L} / \mathrm{mL}$ with PBS) into the nostril I h before injection of OVA into the middle ear. The rats in the OME+Saline group inhaled PBS as the control. (B) The morphology of the tympanic membrane as viewed through an endoscope showing the secretions (arrow) and the radial vascularity (pound sign) (C) The acoustic immittance confirmed the existence of otitis media in the OME group of rats and little otitis media in the OME+IL- $17 \mathrm{mAb}$ group. (D) The statistical analysis of the acoustic immittance according to the compliance values, and ABR measurement was conducted in the rats. The error bars represent the s.e.m. of 5 rats per group. $* \mathrm{P}<0.05,{ }^{\#} \mathrm{P}<0.01$. Data are representative of three independent experiments. 
A

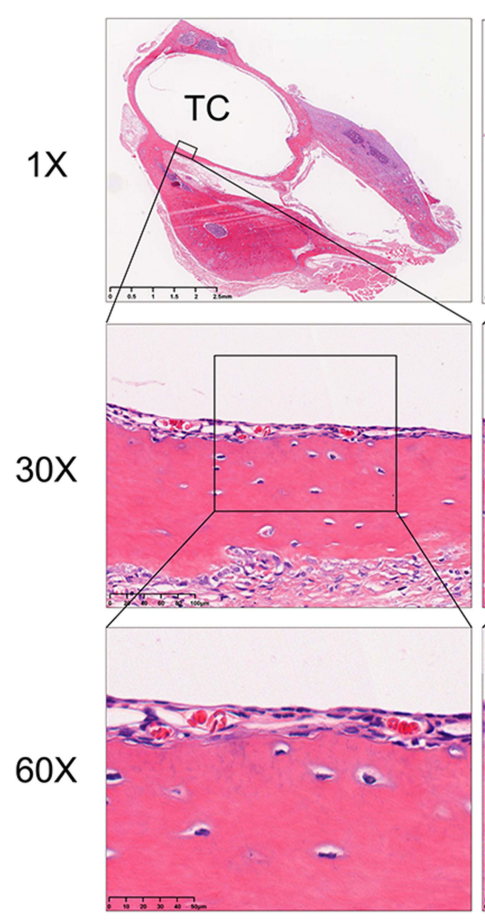

B

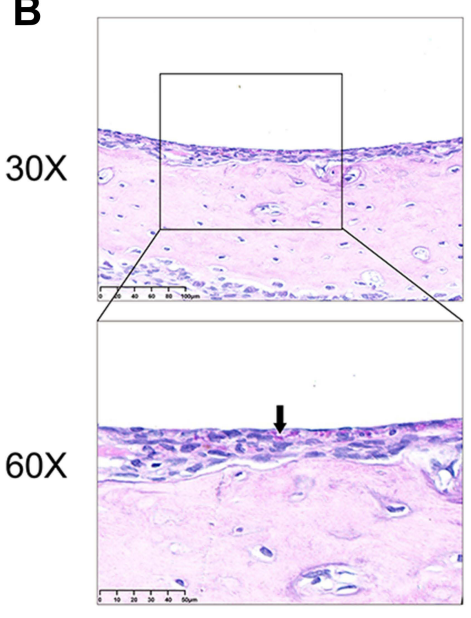

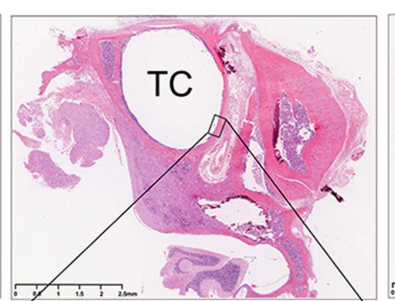
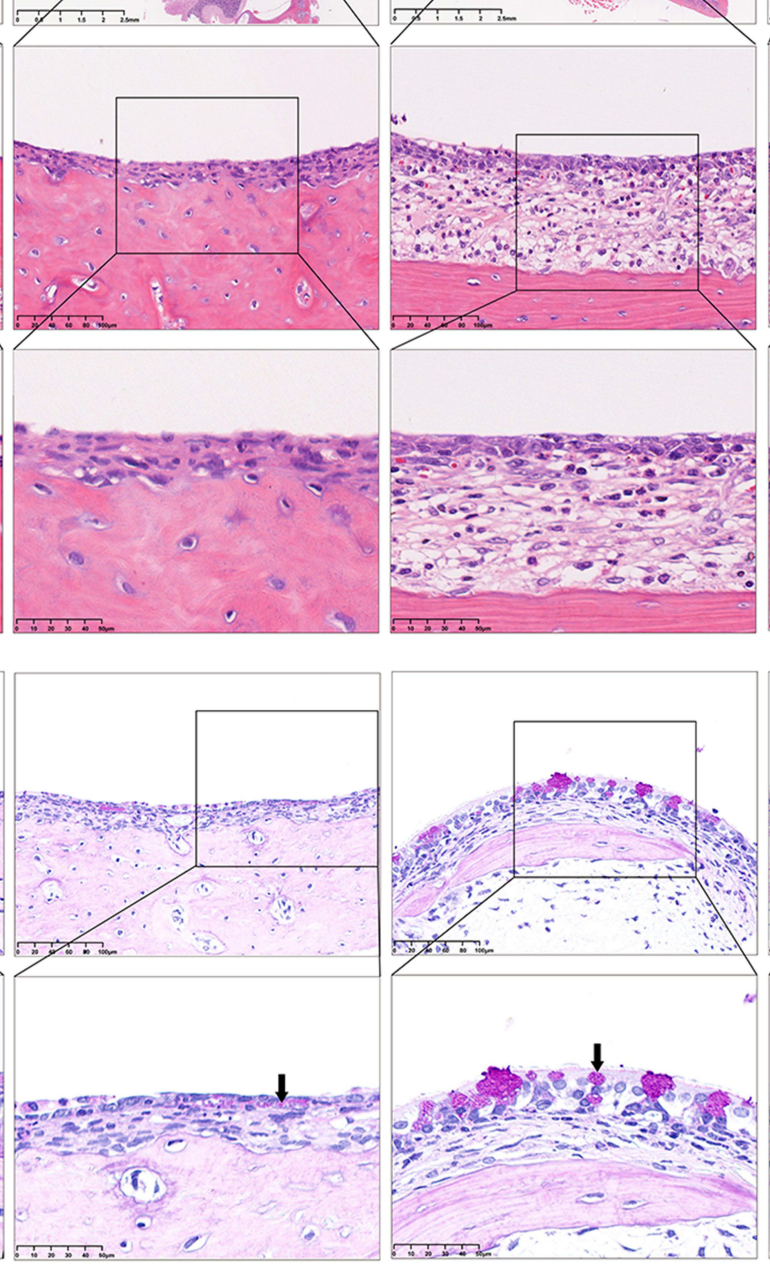

OME + IL-17 mAb
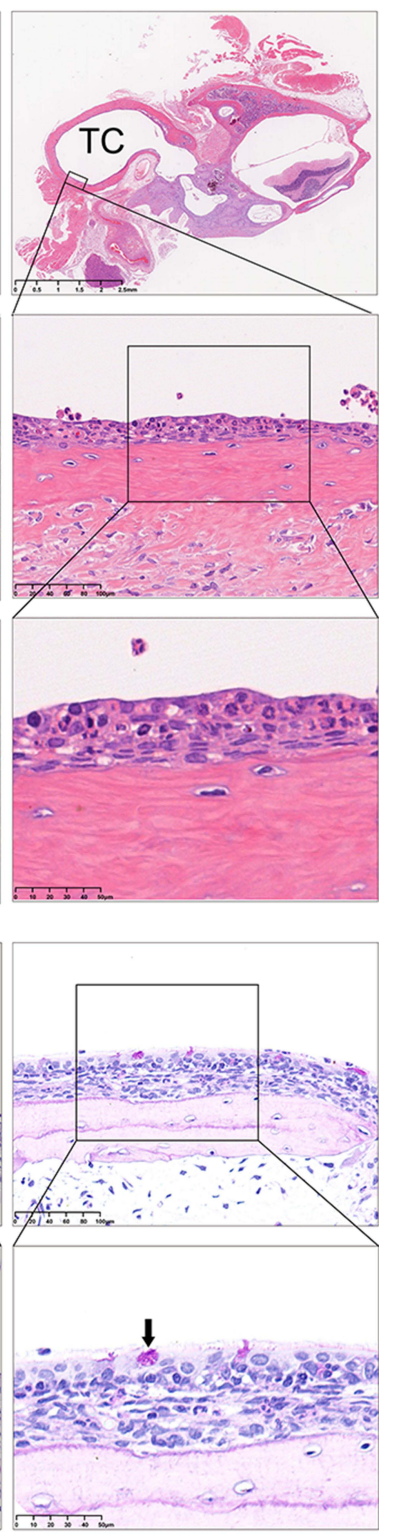

C

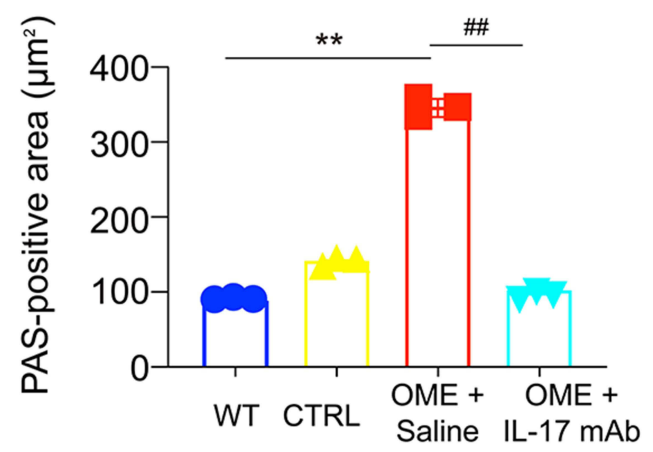

- WT

$\triangle \quad$ CTRL

- OME + Saline

$\checkmark$ OME + IL-17 mAb

Figure $4 \mathrm{IL}-17 \mathrm{mAb}$ ameliorated OME in rats. (A) The severity of the middle ear injury was evaluated by HE staining at Ix, 30x, and $60 \times$ magnification (TC, tympanic cavity). (B) Representative photos are the PAS-stained middle ear sections in different groups. The goblet cells (arrows) and the secretion of mucin was evaluated by PAS staining at $30 \times$ and $60 \times$ magnification. The positivity of PAS staining was purple-red. The statistical analysis of all data from three rats is presented $(\mathbf{C})$. $* * P<0.0$, ${ }^{*} \mathrm{P}<0.0 \mathrm{I}$. Data are representative of three independent experiments. 
hearing loss and alleviated the symptoms of OME in the rats, thus providing evidence for the therapeutic effect of IL-17 in treating OME.

\section{The Anti-IL-I7 mAb Attenuated the IL-I7-Induced Inflammation in OME}

IL-17 plays a key role in the induction of inflammation. To clarify whether the relief of OME was promoted by blocking IL-17, the levels of IL-17 in the blood and MEE were detected 48 hours after nasal drip treatment. The ELISA showed that IL-17 in the serum and MEE was successfully neutralized (Figure 5A), and both the RNA and protein levels of IL-17 in the middle ear tissues were decreased by anti-IL-17 mAb treatment compared to the saline-treated group (Figure 5B and C). Moreover, immunohistochemical staining was used to characterize the IL-17 expression in the middle ear of the rats. IL-17 expression was increased in the middle ear of the OME rats whose tympanic effusion was filled with IL-17 proteins, while the IL-17 levels were decreased in the middle ear of the OME+IL-17 mAb rats (Figure 5D and E). However, anti-IL-17 mAb did not suppress the IL-17 expression of the immune cells from the peripheral blood at the RNA level (Figure 5F), and flow cytometry analysis suggested that the anti-IL-17 mAb had no effect on Th17
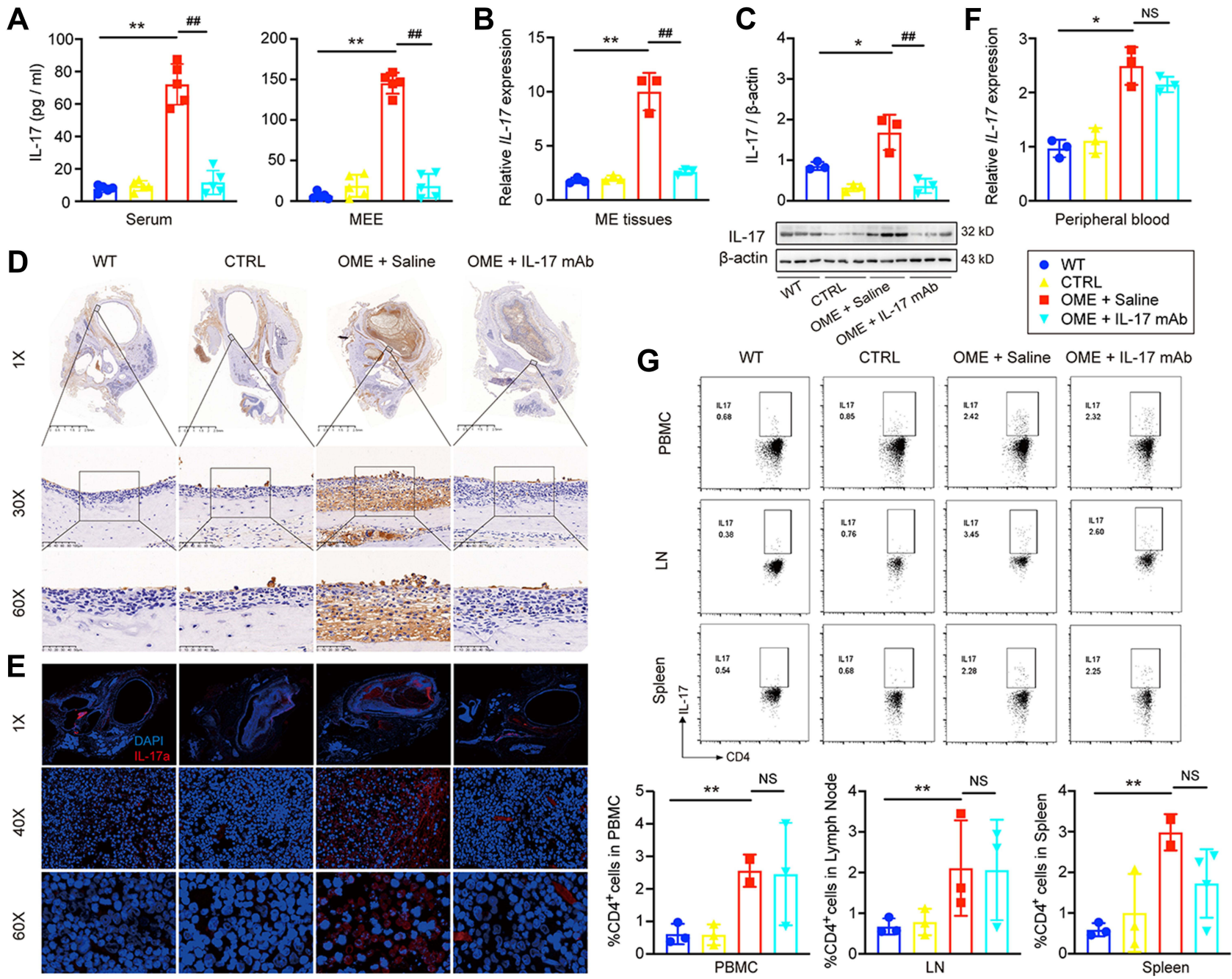

Figure 5 Anti-ILI7 mAb neutralized IL-I7 and attenuated IL-I7-induced inflammation. (A) IL-I7 levels in the serum and MEE were detected by ELISA. The error bars represent the s.e.m. of five rats per group. (B and C) The expression of IL-I7 in the middle ear (ME) tissues was measured by qPCR, Western blot. The error bars represent the s.e.m. of three pairs of ears per group. (D) IL-I7 expression (brown) was determined by the immunohistochemistry at I×, 30×, 60× magnification. (E) IL-I7 expression (brown) was determined by the immunofluorescence analysis at I×, 40×, 60× magnification. IL-I7 (red), DAPI (blue). (F) qPCR was used to measure IL-I7 expression in the immune cells of the peripheral blood. The error bars represent the s.e.m. of three rats per group. (G) Flow cytometry was used to assess the IL-I7 expression of CD4 ${ }^{+}$ T-cells in the peripheral blood mononuclear cell (PBMC), lymph node (LN), and spleen, and the IL-I7 expressing cells were gated from CD4 $4^{+} \mathrm{T}$ cell subset as shown in Figure SI. The error bars represent the s.e.m. of three rats per group. ${ }^{*} \mathrm{P}<0.05,{ }^{* *} \mathrm{P}<0.0 \mathrm{I},{ }^{\#} \mathrm{P}<0.0 \mathrm{I}$. Data are representative of three independent experiments. Abbreviation: NS, not significant. 
differentiation (Figure 5G). Collectively, these results suggested that the relief of OME was due to the blockage of the inflammatory cytokine IL-17 by the mAb.

\section{IL-I7 Induced Inflammatory Responses via the Notch Signaling Pathway}

To further investigate the mechanism by which anti-IL-17 mAb alleviated the OVA-induced middle ear inflammation, the Reactome database was used to analyze the pathways related to the RNA-seq data. The pathway analysis showed the top 20 pathways related to OME and suggested that the Notch signaling pathway is involved in the pathogenesis of OME (Figure 6A). qPCR and Western blot then demonstrated that the expression of Notch1 and its ligand Jagged1 were significantly up-regulated at both the mRNA and protein levels in the middle ear tissues of the OME rats, while they were decreased after the anti-IL-17 mAb treatment (Figure 6B and C), suggesting that the Notch signaling pathway might be activated by IL-17 accumulation in the middle ear in OME. To verify this hypothesis, we used DAPT to suppress the Notch signaling and investigated the effect of DAPT on OME and IL-17 expression. DAPT was injected intraperitoneally $1 \mathrm{~h}$ before the OVA challenge. The expression of Notch1 (Figure 6D) and its ligand Jagged1 (Figure 6E) were suppressed in the middle ear tissues of the OME rats upon DAPT treatment. However, there was no change in IL-17 expression after Notch signaling blockade (Figure 6F). Furthermore, the acoustic immittance diagram of the OME rats upon DAPT treatment showed type A, indicating that there was little otitis media in the middle ear (Figure 6G). These results suggest that the IL-17-induced middle ear inflammation associated with OME is mediated through the Notch signaling pathway.

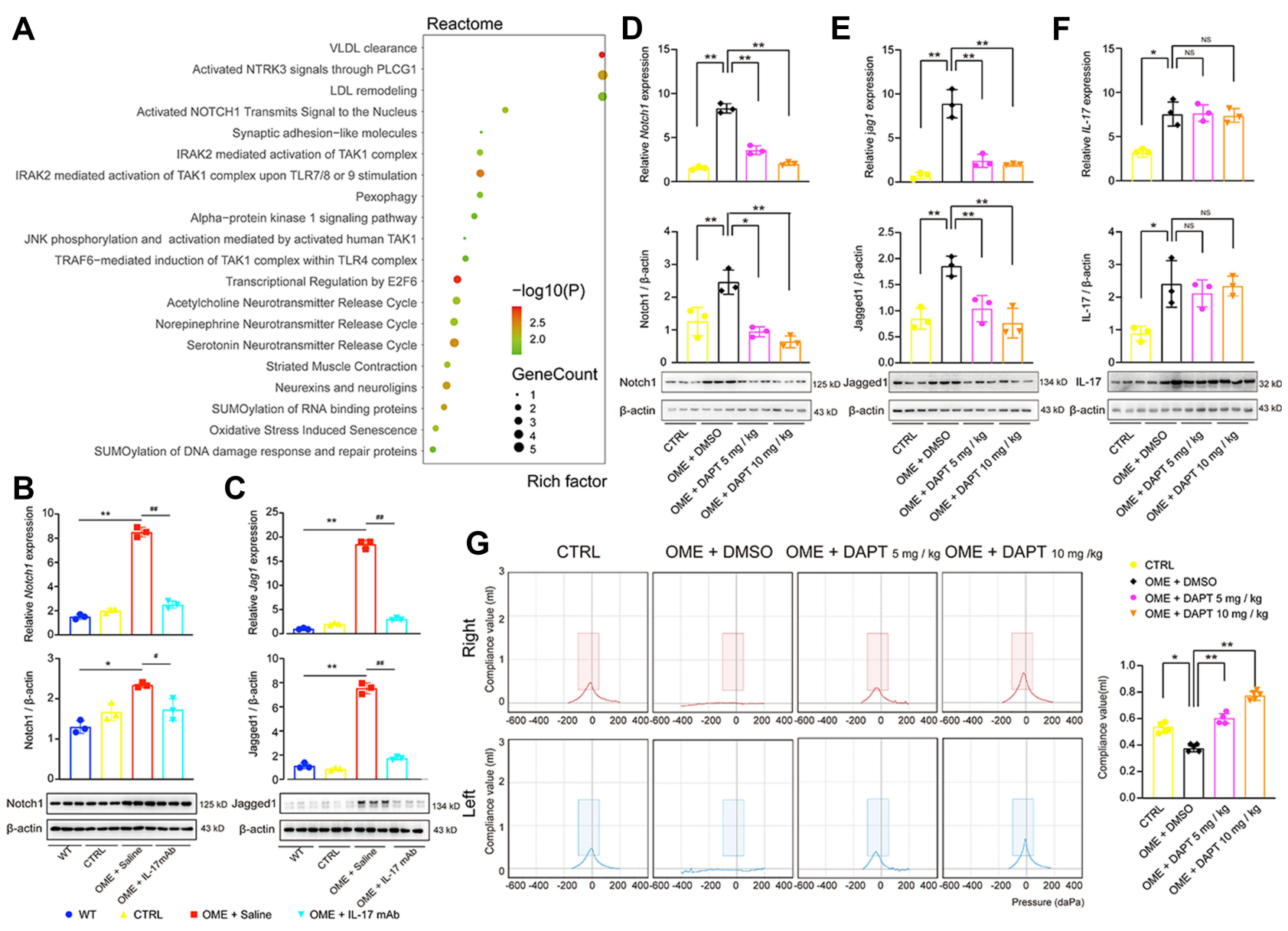

Figure 6 IL- 17 induced inflammatory responses in the middle ear via the Notch signaling pathway. (A) Pathway analysis using the Reactome database showed the top 20 pathways related to OME. (B-F) qPCR and Western blot were used to detect the mRNA expression and protein expression of Notch I, Jagged I, and IL-I7 in the rat middle ear tissue. The error bars represent the s.e.m. of three pairs of ears per group. (G) The acoustic immittance confirmed little otitis media in the OME+DAPT group. The error bars represent the s.e.m. of five ears per group. ${ }^{*} \mathrm{P}<0.05,{ }^{*} \mathrm{P} P<0.01,{ }^{\#} \mathrm{P}<0.05,{ }^{\# \#} \mathrm{P}<0.01$. Data are representative of three independent experiments. Abbreviation: ns, not significant. 


\section{Discussion}

In this study, we investigated the therapeutic effects of an anti-IL-17 mAb on OME. OME is a non-suppurative inflammatory disease of the middle ear that is characterized by MEE and hearing loss. In this study, we first established the OME model in rats through sensitization with intraperitoneal injection of OVA followed by immune challenge by OVA in the middle ear. RNA-seq data suggested that the IL-17 signaling pathway might play a key role in OME pathogenesis, and IL-17 expression was confirmed to be increased both in the serum and in the middle ear in the rat model. Administration of anti-IL-17 mAb inhibited the inflammation in the middle ear and ameliorated the symptoms of OME. Finally, our mechanistic study suggested that IL-17 induced the activation of the Notch signaling pathway to enhance inflammation in the middle ear. Taken together, these findings suggested a new mechanism for OME pathogenesis and suggested an effective approach for OME therapy.

Previous studies have reported that allergic reactions are involved in the development of OME. ${ }^{33}$ The mucosa of the middle ear derives from the same ectoderm as the upper respiratory tract epithelium, suggesting that the middle ear mucosa and upper respiratory epithelium have similar immune functions. ${ }^{1}$ More recent studies have shown that the occurrence and progression of OME are closely related to immune factors. ${ }^{26}$ For example, neutrophils are involved in type 1 allergic reactions and exacerbate the inflammation of the middle ear, and during the initiation of OME antigen-presenting cells in the draining lymph nodes are activated and produce the cytokines needed to regulate the differentiation and proliferation of $\mathrm{CD}^{+} \mathrm{T}$ helper (Th) cells such as the Th1 cells, Th2 cells, and Th17 cells. ${ }^{15} \mathrm{Th} 1$ cells produce IFN- $\gamma$ and Th2 cells are functionally defined by the production of IL-4, while Th17 cells produce IL-17. ${ }^{34}$ In the present study, we found that the rats in the OME group showed increased inflammatory infiltration and an increased number of goblet cells compared to rats in the control (CTRL) group (Figure 4). IL-17 ${ }^{+}$Th17 cells were identified in the middle ear tissue, suggesting that OME-related inflammation in the middle ear was induced by the pro-inflammatory cytokine IL-17 (Figure 5D and E).

Cytokines have been shown to play essential roles in OME. The production of Th2 cytokines was reported to be increased in the middle ear mucosa of the OVA-induced OME animal model, while the expression of Th1 cytokines was decreased. ${ }^{24}$ Yeghaneh et al showed that the IL-17 levels in the serum of OME patients were significantly higher than controls, suggesting that IL-17 is a pro-inflammatory cytokine in OME. ${ }^{28}$ In this study, we identified IL-17 as a key regulator in the pathogenesis of OME. IL-17 is a pro-inflammatory cytokine that induces the expression of other cytokines and chemokines, and it recruits neutrophils to sites of inflammation. The Th17 and IL-17 signaling pathways have been demonstrated to play a vital role in many inflammatory diseases and autoimmune diseases. ${ }^{35}$ Our RNA-seq results showed that IL-17 signaling was activated in the middle ear of the OME rats (Figure 2A and B), and the ELISA analysis demonstrated that the IL-17 levels were significantly increased in the serum and MEE of the OME rats (Figure 2C and D). Moreover, the immunohistochemical staining and immunofluorescence analysis showed the presence of IL-17 in the middle ear of the OME rats (Figure 5D and E). In clinical studies of autoimmune diseases, the expression of IL-17 was found to be up-regulated in the mouse model of psoriasis, and knockout of $I L-17 R A$ significantly improves the abnormal proliferation of the stratum corneum of the skin. ${ }^{36,37}$ The humanized anti-IL-17A monoclonal antibody Secukinumab has been clinically tested in rheumatoid arthritis patients and has been shown to induce a rapid and longlasting response, ${ }^{38}$ and Brodalumab, a human mAb developed by AstraZeneca to block IL-17RA, has also begun to be used in the treatment of rheumatoid arthritis. ${ }^{39}$ Our study applied the anti-IL-17 mAb to OME treatment and found that the anti-IL-17 mAb suppressed the inflammation in the middle ear (Figure 4A) and significantly relieved the symptoms of OME (Figure 3B and C). Our study provides evidence for anti-IL-17 as an effective approach to OME therapy, but further studies are still required to elucidate the molecular mechanisms by which IL-17 promotes middle ear inflammation in OME.

During the pathogenesis of OME, the goblet cells secrete a large amount of mucin. The PAS staining showed that the mucin was stained purple-red due to glycoprotein-containing cytoplasm in the OME group, and the majority of goblet cells in the OME group indicated the hyperplasia of epithelial goblet cells and their mucus secretion (Figure 4B). The Notch signaling pathway can promote mucous cell proliferation and mucus secretion, and when the Notch signaling pathway is blocked mucus secretion is significantly reduced. ${ }^{40}$ We verified that the Notch signaling pathway was activated in the OME group and was suppressed by blockage of IL-17 (Figure 6), suggesting that the Notch pathway is involved in the mucus secretion. Muc5ac is mainly secreted by goblet cells, and Notch signaling is considered to be the 
main regulator of goblet cell secretion. ${ }^{41}$ Further studies are required to determine the detailed molecular mechanism by which IL-17 promotes Notch1 activation and regulates the expression of Muc5ac.

Taken together, our study showed that IL-17 induces middle ear inflammation via the Notch signaling pathway in OVA-induced allergic OME, and this could be ameliorated upon anti-IL-17 mAb treatment in vivo, thus providing evidence for targeting IL-17 as a novel therapeutic intervention in OME.

\section{Acknowledgments}

This work was supported by the National Natural Science Foundation of China (Nos. 82071055, 81970879, 82192860), the Excellent Doctors-Excellent Clinical Researchers Program (No. SZA202002), and the Shanghai Clinical Center for Disease of Otorhinolaryngology Project (20MC1920200, 21Y31900500) and Research Projects of the Shanghai Municipal Health Committee (2020YJZX0110, 21Y31900501).

\section{Author Contributions}

All authors made a significant contribution to the work reported, whether that is in the conception, study design, execution, acquisition of data, analysis and interpretation, or in all these areas; took part in drafting, revising or critically reviewing the article; gave final approval of the version to be published; have agreed on the journal to which the article has been submitted; and agree to be accountable for all aspects of the work.

\section{Disclosure}

The authors declare that no conflict of interest exists.

\section{References}

1. Schilder AG, Chonmaitree T, Cripps AW, et al. Otitis media. Nat Rev Dis Primers. 2016;2:16063. doi:10.1038/nrdp.2016.63

2. Monasta L, Ronfani L, Marchetti F, et al. Burden of disease caused by otitis media: systematic review and global estimates. PLoS One. 2012;7: e36226. doi:10.1371/journal.pone.0036226

3. Pichichero ME. Helping children with hearing loss from otitis media with effusion. Lancet. 2018;392:533-534. doi:10.1016/S0140-6736(18) $31862-2$

4. Rosenfeld RM, Shin JJ, Schwartz SR, et al. Clinical practice guideline: otitis media with effusion (update). Otolaryngol Head Neck Surg. 2016;154: S1-S41. doi:10.1177/0194599815623467

5. Rovers MM, Schilder AG, Zielhuis GA, Rosenfeld RM. Otitis media. Lancet. 2004;363:465-473. doi:10.1016/S0140-6736(04)15495-0

6. Thompson PL, Gilbert RE, Long PF, et al. Effect of antibiotics for otitis media on mastoiditis in children: a retrospective cohort study using the United Kingdom general practice research database. Pediatrics. 2009;123:424-430. doi:10.1542/peds.2007-3349

7. Chonmaitree T, Revai K, Grady JJ, et al. Viral upper respiratory tract infection and otitis media complication in young children. Clin Infect Dis. 2008;46:815-823. doi:10.1086/528685

8. Waldron MN, Matthews JN, Johnson IJ. The effect of otitis media with effusions on balance in children. Clin Otolaryngol Allied Sci. 2004;29:318-320. doi:10.1111/j.1365-2273.2004.00833.x

9. Cohen H, Friedman EM, Lai D, et al. Balance in children with otitis media with effusion. Int J Pediatr Otorhinolaryngol. 1997;42:107-115. doi:10.1016/s0165-5876(97)00113-4

10. Casselbrant ML, Villardo RJ, Mandel EM. Balance and otitis media with effusion. Int J Audiol. 2008;47:584-589. doi:10.1080/ 14992020802331230

11. Bennett KE, Haggard MP, Silva PA, Stewart IA. Behaviour and developmental effects of otitis media with effusion into the teens. Arch Dis Child. 2001;85:91-95. doi:10.1136/adc.85.2.91

12. Elicora SS, Öztürk M, Sevinç R, et al. Risk factors for otitis media effusion in children who have adenoid hypertrophia. Int $J$ Pediatr Otorhinolaryngol. 2015;79:374-377. doi:10.1016/j.ijporl.2014.12.030

13. Engel J, Anteunis L, Volovics A, Hendriks J, Marres E. Risk factors of otitis media with effusion during infancy. Int J Pediatr Otorhinolaryngol. 1999;48:239-249. doi:10.1016/s0165-5876(99)00037-3

14. Kuo CL, Lien CF, Chu CH, Shiao AS. Otitis media with effusion in children with cleft lip and palate: a narrative review. Int $J$ Pediatr Otorhinolaryngol. 2013;77:1403-1409. doi:10.1016/j.ijporl.2013.07.015

15. Mittal R, Kodiyan J, Gerring R, et al. Role of innate immunity in the pathogenesis of otitis media. Int J Infect Dis. 2014;29:259-267. doi:10.1016/j. ijid.2014.10.015

16. Chantzi FM, Kafetzis DA, Bairamis T, et al. IgE sensitization, respiratory allergy symptoms, and heritability independently increase the risk of otitis media with effusion. Allergy. 2006;61:332-336. doi:10.1111/j.1398-9995.2006.00971.x

17. Daniel M, Imtiaz-Umer S, Fergie N, Birchall JP, Bayston R. Bacterial involvement in otitis media with effusion. Int J Pediatr Otorhinolaryngol. 2012;76:1416-1422. doi:10.1016/j.ijporl.2012.06.013

18. Tawfik SA, Ibrahim AA, Talaat IM, El-Alkamy SS, Youssef A. Role of bacterial biofilm in development of middle ear effusion. Eur Arch OtoRhino-L. 2016;273:4003-4009. doi:10.1007/s00405-016-4094-2 
19. Christensen ED, Thorsen J, Stokholm J, et al. Early life bacterial airway colonization, local immune mediator response and risk of otitis media. J Med Microbiol. 2020;69:1124-1131. doi:10.1099/jmm.0.001227

20. Leichtle A, Lai Y, Wollenberg B, Wasserman SI, Ryan AF. Innate signaling in otitis media: pathogenesis and recovery. Curr Allergy Asthma Rep. 2011;11:78-84. doi:10.1007/s11882-010-0158-3

21. MacIntyre EA, Chen C-M, Herbarth O, et al. Early-life otitis media and incident atopic disease at school age in a birth cohort. Pediatr Infect Dis J. 2010;29:e96-99. doi:10.1097/inf.0b013e3181fcd9e8

22. MacIntyre EA, Heinrich J. Otitis media in infancy and the development of asthma and atopic disease. Curr Allergy Asthma Rep. 2012;12:547-550. doi: $10.1007 / \mathrm{s} 11882-012-0308-\mathrm{X}$

23. Mittal R, Robalino G, Gerring R, et al. Immunity genes and susceptibility to otitis media: a comprehensive review. $J$ Genet Genomics. 2014;41:567-581. doi:10.1016/j.jgg.2014.10.003

24. Kariya S, Okano M, Hattori H, et al. TH1/TH2 and regulatory cytokines in adults with otitis media with effusion. Otol Neurotol. 2006;27:1089-1093. doi:10.1097/01.mao.0000224087.93096.4d

25. Smirnova MG, Birchall JP, Pearson JP. Evidence of T-helper cell 2 cytokine regulation of chronic otitis media with effusion. Acta Otolaryngol. 2005;125:1043-1050. doi:10.1080/00016480510035449

26. Enoksson F, Ruiz Rodriguez A, Peno C, et al. Niche- and gender-dependent immune reactions in relation to the microbiota profile in pediatric patients with otitis media with effusion. Infect Immun. 2020;88. doi:10.1128/IAI.00147-20

27. Zielnik-Jurkiewicz B, Stankiewicz-Szymczak W. Pro-inflammatory interleukins in middle ear effusions from atopic and non-atopic children with chronic otitis media with effusion. Eur Arch Oto-Rhino-L. 2016;273:1369-1378. doi:10.1007/s00405-015-3683-9

28. Yeghaneh Moghaddam A, Talaei R, Nikoueinejad H, Akbari H. Studying the serum as well as serous level of il-17 and il-23 in patients with serous otitis media. Iran J Allergy Asthma Immunol. 2017;16:520-524.

29. Krueger A, Val S, Pérez-Losada M, et al. Relationship of the middle ear effusion microbiome to secretory mucin production in pediatric patients with chronic otitis media. Pediatr Infect Dis J. 2017;36:635-640. doi:10.1097/INF.0000000000001493

30. Kang JH, Lee EH, Park SW, Chung IY. MUC5AC expression through bidirectional communication of Notch and epidermal growth factor receptor pathways. J Immunol. 2011;187:222-229. doi:10.4049/jimmunol.1003606

31. Bernstein J. Biological mediators of inflammation in middle ear effusions. Ann Otol Rhinol Laryngol. 1976;85:90-96. doi:10.1177/ $00034894760850 \mathrm{~S} 218$

32. Hardy SM, Heavner SB, White DR, et al. Late-phase allergy and eustachian tube dysfunction. Otolaryngol Head Neck Surg. 2001;125:339-345. doi:10.1067/mhn.2001.119140

33. Francis NA, Cannings-John R, Waldron C-A, et al. Oral steroids for resolution of otitis media with effusion in children (OSTRICH): a double-blinded, placebo-controlled randomised trial. Lancet. 2018;392:557-568. doi:10.1016/S0140-6736(18)31490-9

34. Borst J, Ahrends T, Babala N, Melief CJM, Kastenmuller W. CD4(+) T cell help in cancer immunology and immunotherapy. Nat Rev Immunol. 2018;18:635-647. doi:10.1038/s41577-018-0044-0

35. Gaffen SL, Jain R, Garg AV, Cua DJ. The IL-23-IL-17 immune axis: from mechanisms to therapeutic testing. Nat Rev Immunol. 2014;14:585-600. doi:10.1038/nri3707

36. Johansen C, Usher PA, Kjellerup RB, et al. Characterization of the interleukin-17 isoforms and receptors in lesional psoriatic skin. Br J Dermatol. 2009;160:319-324. doi:10.1111/j.1365-2133.2008.08902.x

37. Yamaguchi Y, Fujio K, Shoda H, et al. IL-17B and IL-17C are associated with TNF-alpha production and contribute to the exacerbation of inflammatory arthritis. J Immunol. 2007;179:7128-7136. doi:10.4049/jimmunol.179.10.7128

38. Papp KA, Weinberg MA, Morris A, Reich K. IL17A/F nanobody sonelokimab in patients with plaque psoriasis: a multicentre, randomised, placebo-controlled, phase 2b study. Lancet. 2021;397:1564-1575. doi:10.1016/S0140-6736(21)00440-2

39. Nirula A, Nilsen J, Klekotka P, et al. Effect of IL-17 receptor A blockade with brodalumab in inflammatory diseases. Rheumatology. 2016;55:ii43ii55. doi:10.1093/rheumatology/kew346

40. Christopoulos PF, Gjølberg TT, Krüger S, et al. Targeting the notch signaling pathway in chronic inflammatory diseases. Front Immunol. 2021;12:668207. doi:10.3389/fimmu.2021.668207

41. Song X, He X, Li X, Qian Y. The roles and functional mechanisms of interleukin-17 family cytokines in mucosal immunity. Cell Mol Immunol. 2016;13:418-431. doi:10.1038/cmi.2015.105

Journal of Inflammation Research

\section{Publish your work in this journal}

The Journal of Inflammation Research is an international, peer-reviewed open-access journal that welcomes laboratory and clinical findings on the molecular basis, cell biology and pharmacology of inflammation including original research, reviews, symposium reports, hypothesis formation and commentaries on: acute/chronic inflammation; mediators of inflammation; cellular processes; molecular mechanisms; pharmacology and novel anti-inflammatory drugs; clinical conditions involving inflammation. The manuscript management system is completely online and includes a very quick and fair peer-review system. Visit http://www.dovepress.com/testimonials.php to read real quotes from published authors.

Submit your manuscript here: https://www.dovepress.com/journal-of-inflammation-research-journal 